\title{
GRAIN SIZE DEPENDENCE OF THE GAUGE FACTOR OF THIN METALLIC FILMS
}

\author{
C. R. TELLIER and A. J. TOSSER \\ Université de Nancy I, Laboratoire d'Electronique, Case officielle $n^{\circ}$ 140, 54037 - Nancy - Cedex, France
}

(Received May 10, 1976; in final form April 5, 1977)

\begin{abstract}
The combined effects of grain boundary, external surface and background scattering (Mayadas and Shatzkes model) are considered. Theoretical expressions of the transverse and longitudinal strain coefficient of resistance of monocrystalline and polycrystalline films are calculated. These general formulae agree with those previously proposed for infinitely thick polycrystalline films.
\end{abstract}

\section{INTRODUCTION}

Several sets of experimental data on the strain dependence of the electrical resistivity of thin conductive $^{1-2}$ or thin metallic ${ }^{3-6}$ films have been reported in the past few years. The theoretical aspects of the changes in electrical resistance with applied strain have been extensively investigated ${ }^{1-4,6-12}$ for thin continuous films whose resitivity exhibit FuchsSondheimer ${ }^{13}$ size effect.

Recently it has been observed that in thin evaporated metal films, the grains have a columnar shape ${ }^{14-16}$ and that the grain-boundaries effect on film resistivity cannot be considered as negligible. Hence, in order to interpret their experimental data on aluminum films, Mayadas ans Shatzkes ${ }^{17}$ have proposed a conduction model (M-S model) which takes into account three types of electron scattering mechanisms: an isotropic background scattering, a grain boundary scattering and an external surface scattering. The operative grain-boundaries can be represented by a series of randomly spaced partially reflecting planes, perpendicular to the electric field, whose normals lie in the substrate surface; if the interplanar spacing $d$ is identified with the average grain diameter $D$, the film conductivity $\sigma_{f}$ is given by

$$
\sigma_{f}=\sigma_{b}[F(\alpha)-A]
$$

Here, $\sigma_{b}$ is the bulk conductivity (i.e. the conductivity of an infinitely thick monocrystalline film)

$$
\begin{aligned}
& A=\frac{6}{\pi k}(1-p) \int_{0}^{\pi / 2} d \phi \int_{1}^{\infty} d \tau \\
& \frac{\cos ^{2} \phi}{H^{2}(\tau, \phi)}\left(\frac{1}{\tau^{3}}-\frac{1}{\tau^{5}}\right) \frac{1-\exp \{-k \tau H(\tau, \phi)\}}{1-p \exp \{-k \tau H(\tau, \phi)\}} \\
& H(\tau, \phi)=1+\alpha(\cos \phi)^{-1}\left[1-1 / \tau^{2}\right]^{-1 / 2}
\end{aligned}
$$

and

$$
F(\alpha)=1-\frac{3}{2} \alpha+3 \alpha^{2}-3 \alpha^{3} \ln \left(1+\frac{1}{\alpha}\right)
$$

The $\alpha$ parameter is related to the bulk mean free path $\lambda$, to the average grain diameter $D$ and to the grain boundary reflection coefficient, $r$, by equation (1.4)

$$
\alpha=\frac{\lambda}{D} \cdot \frac{r}{1-r}
$$

The $\mathrm{M}-\mathrm{S}$ model is particularly relevant to description of the thickness dependence of both resistivity and its temperature coefficient for thin sputtered metal films; ${ }^{1-19}$ we have previously shown ${ }^{18-20}$ that such sputtered films exhibit, according to the twolayers model, ${ }^{21-23}$ a fine-grained structure defined by a constant average grain size. ${ }^{18-20}$

Taking the M-S model into account, B. S. Verma et.al. ${ }^{11}$ and Awatar Singh ${ }^{12}$ have recently published theoretical studies of the strain coefficient of 
resistivity of thin polycrystalline metal films in two special cases. In the first case, ${ }^{11}$ it has been assumed that the electron scattering from external surface can be neglected (i.e. the authors have only calculated the strain gauge factor of infinitely thick polycry. stalline films); in the second case a simplified equation, derived from the well-known FuchsSondheimer relation ${ }^{13}$ has been used to express the film conductivity in the presence of both grain boundaries and external surfaces scatterings.

However, no general theoretical relations have been derived for the strain gauge factor of thin continuous polycrystalline films whose total resistivity is given by the general $\mathrm{M}-\mathrm{S}$ relation Eq. (1).

It is the purpose of this paper to calculate the longitudinal and transverse strain coefficient of resistance of thin polycrystalline films by including the influence of the substrate elasticity on the strain coefficient of films. When the ratio $k$ between the film thickness $t$ and the bulk mean free path $\lambda$ becomes high (i.e. $k$ becomes infinite) the deduced general expressions reduce to those derived by B.S. Verma et al. ${ }^{11}$ and Awatar Singh ${ }^{12}$ for infinitely thick self supporting polycrystalline films.

\section{THEORETICAL TREATMENT}

\subsection{General Relations}

The electrical resistance $R_{f}$ of the film is related to the film conductivity $\sigma_{f}$ by Eq. (2)

$$
R_{f}=\frac{1}{\sigma_{f}} \cdot \frac{L}{w t}
$$

Where, $L, w$ and $t$ are the film length width and thickness respectively.

A logarithmic differentiation of Eq. (2) gives

$$
\frac{d R_{f}}{R_{f}}=\frac{d L}{L}-\frac{d \sigma f}{\sigma_{f}}-\frac{d w}{w}-\frac{d t}{t}
$$

When a transverse strain $d w / w$ is applied to the substrate, a longitudinal strain $d L / L=-\mu_{s}(d w / w)$ will occur where $\mu_{s}$ is the Poisson's ratio of the substrate material. The transverse and longitudinal stresses are transmitted to the film, but the thickness direction in the film is stress free. Hence, the wellknown elasticity formulae lead to the strain equations

$$
\frac{d t}{t}=-\mu \frac{\left(1-\mu_{s}\right)}{(1-\mu)} \frac{d w}{w}
$$

where $\mu$ is the Poisson's ratio of the film material.
The transverse strain coefficient of resistance of the film $\gamma_{f T}$, which is also called the transverse gauge factor, is defined as

$$
\gamma_{f T}=\left(\frac{d R_{f}}{R_{f}}\right) /\left(\frac{d w}{w}\right)
$$

Dividing equation (3) by $\frac{d w}{w}$ gives

$$
\gamma_{f T}=-1-\mu_{s}+\frac{\mu\left(1-\mu_{s}\right)}{1-\mu}-\left(\frac{d \sigma_{f}}{\sigma_{f}}\right) /\left(\frac{d w}{w}\right)
$$

If thick films are considered as unsupported films, we can calculate the bulk value of the transverse gauge factor by substituting $\sigma_{f}$ by $\sigma_{b}$ (bulk conductivity) and $\mu_{s}$ by $\mu$ in Eq. (6), hence

$$
\gamma_{b T}=-1-\left(\frac{d \sigma_{b}}{\sigma_{b}}\right) /\left(\frac{d w}{w}\right)
$$

With the assumption that the variation of $\sigma_{b}$ and $\lambda$ with $w$ may be entirely attributed to the change in amplitude of the thermal vibrations of atoms with applied strain, the strain coefficient of $\sigma_{b}$ and $\lambda$ are expressed as Eq. (4).

$$
\begin{aligned}
& {\left[\frac{d \sigma_{b}}{\sigma_{b}}\right] /\left[\frac{d w}{w}\right]=2 g[2 \mu-1]-1} \\
& {\left[\frac{d \lambda}{\lambda}\right] /\left[\frac{d w}{w}\right]=2 g[2 \mu-1]}
\end{aligned}
$$

where $g$ is the Grüneisen 's constant.

From Eq. (7) we deduce

$$
\gamma_{b T}=2 g(1-2 \mu)
$$

In a similar way, when a longitudinal strain $d L / L$ is applied to the substrate, the strain equations can be written

$$
\begin{aligned}
& \frac{d w}{w}=-\mu_{s} \frac{d L}{L} \\
& \frac{d t}{t}=-\mu \frac{\left(1-\mu_{s}\right)}{(1-\mu)} \frac{d L}{L}
\end{aligned}
$$

Hence, the longitudinal gauge factor defined as

$$
\gamma_{f L}=\left(\frac{d R_{f}}{R_{f}}\right) /\left(\frac{d L}{L}\right)
$$

can be expressed by

$$
\gamma_{f L}=1+\mu_{s}+\mu \frac{\left(1-\mu_{s}\right)}{(1-\mu)}-\left(\frac{d \sigma_{f}}{\sigma_{f}}\right) /\left(\frac{d L}{L}\right)
$$


Therefore, the bulk value of the longitudinal gauge factor is

$$
\gamma_{b L}=2(\mu+1)+2 g[1-2 \mu]
$$

\subsection{The $M-S$ Model}

In the Mayadas-Shatzkes model two different types of thin metal films may be considered: ${ }^{16,24,35,26}$ the polycrystalline films whose grain size $D$ is constant and thickness independent and the monocrystalline films whose grain size is equal to thickness.

2.2.1 The electrical conductivity When the substrate is bent to produce a longitudinal (or a transverse) strain, it is reasonable to expect variations in length $L$, width $w$, thickness $t$ and grain size diameter $D$ of the film; at the same time the strain introduces a change in the bulk mean free path $\lambda$ and the bulk conductivity $\sigma_{b}$; the grain boundary electronic reflection coefficient $r$ is considered as a parameter.

Let us note that the interplanar spacing $d$ which lies in the direction of the applied electric field (i.e. in the length $L$ direction) is identified as the average grain diameter $D$.

Hence, $\alpha$ and $\sigma_{f}$ are the functions

$$
\alpha=\alpha(\lambda, D, r)
$$

and

$$
\sigma_{f}=\sigma_{f}\left(\sigma_{b}, \lambda, D, t, r\right)
$$

with the assumption that $\frac{d D}{D} \approx \frac{d L}{L}$

The differential of $\sigma_{f}$ can thus be expressed as

$$
\begin{aligned}
d \sigma_{f}=\frac{\partial \sigma_{f}}{\partial \sigma_{b}} d \sigma_{b}+\frac{\partial \sigma_{f}}{\partial \lambda} d \lambda+ & \frac{\partial \sigma_{f}}{\partial D} d D \\
& +\frac{\partial \sigma_{f}}{\partial t} d t+\frac{\partial \sigma_{f}}{\partial r} d r
\end{aligned}
$$

Introducing Eq. (1) into Eq. (16) then dividing by $\sigma_{f}$, we obtain

$$
\begin{aligned}
& \frac{d \sigma_{f}}{\sigma_{f}}=\frac{d \sigma_{b}}{\sigma_{b}}+\frac{1}{F(\alpha)-A}\left[\left\{\frac{\partial F}{\partial \lambda} d \lambda+\frac{\partial F}{\partial D} d D\right.\right. \\
& \left.+\frac{\partial F}{\partial r} d r\right\}-\left\{\frac{\partial A}{\partial \lambda} d \lambda+\frac{\partial A}{\partial D} d D+\frac{\partial A}{\partial t} d t\right. \\
& \left.\left.+\frac{\partial A}{\partial r} d r\right\}\right]
\end{aligned}
$$

by solving the derivatives we get

$$
\begin{aligned}
& \frac{d \sigma_{f}}{\sigma_{f}}=\frac{d \sigma_{b}}{\sigma_{b}}+\frac{1}{F(\alpha)-A}\left\{X\left[\frac{d \lambda}{\lambda}-\frac{d D}{D}\right]\right. \\
& \left.+Y\left[\frac{d D}{D}-\frac{d t}{t}\right]+\frac{X-Y}{1-r} \cdot \frac{d r}{r}\right\}
\end{aligned}
$$

where

$$
X=G(\alpha)+U
$$

with

$$
\begin{aligned}
& G(\alpha)=\alpha \frac{\partial E}{\partial \alpha}=-\frac{3}{2} \alpha+6 \alpha^{2}+\frac{3 \alpha^{3}}{1+\alpha} \\
& -9 \alpha^{3} 1 n\left(1+\frac{1}{\alpha}\right) \\
& U=\frac{6}{\pi k}(1-p) \int_{0}^{\pi / 2} d \phi \int_{1}^{\infty} d r \frac{\cos ^{2} \phi}{H^{2}(\tau, \phi)}\left[\frac{1}{\tau^{3}} \frac{1}{\tau^{5}}\right] \\
& \left(1-2 H^{-1}(\tau, \phi)\right) \frac{1-\exp \{-k \tau H(\tau, \phi)\}}{1-p \exp \{-k \tau H(\tau, \phi)\}} \\
& +\frac{6}{\pi}(1-p)^{2} \int_{0}^{\pi / 2} d \phi \int_{1}^{\infty} d \tau \\
& \frac{\cos ^{2} \phi}{H^{2}(\tau, \phi)}\left[\frac{1}{\tau^{2}}-\frac{1}{\tau^{4}}\right] \frac{\exp \{-k \tau H(\tau, \phi)\}}{[1-p \exp \{-k \tau H(\tau, \phi)\}]^{2}}
\end{aligned}
$$

$$
\begin{aligned}
Y= & \frac{6}{\pi}(1-p)^{2} \int_{o}^{\pi / 2} d \phi \int_{1}^{\infty} d \tau \frac{\cos ^{2} \phi}{H(\tau, \phi)}\left[\frac{1}{\tau^{2}}-\frac{1}{\tau^{4}}\right] \\
& \frac{\exp \{-k \tau H(\tau, \phi)\}}{[1-p \exp \{-k \tau H(\tau, \phi)\}]^{2}} \\
& -\frac{6}{\pi k}(1-p) \int_{o}^{\pi / 2} d \phi \int_{1}^{\infty} d \tau \frac{\cos ^{2} \phi}{H^{2}(\tau, \phi)}\left[\frac{1}{\tau^{3}}-\frac{1}{\tau^{5}}\right] \\
& \left.\frac{1-\exp \{-k \tau H(\tau, \phi)\}}{1-p \exp \{-k \tau H(\tau, \phi)}\right\}
\end{aligned}
$$

2.2.2 The longitudinal gauge factor Taking into account the influence of the substrate elasticity on the gauge factor of the film, we can write

$$
\frac{d \lambda}{\lambda}=-\eta \frac{d L}{L}
$$




$$
\begin{aligned}
& \frac{d t}{t}=-\mu \frac{\left(1-\mu_{s}\right)}{(1-\mu)} \frac{d L}{L} \\
& \frac{d D}{D} \approx \frac{d L}{L}
\end{aligned}
$$

In these equations we use

$$
\eta=2 g(1-2 \mu)
$$

Hence, the calculations lead to Eq. (20) assuming the strain coefficient of grain boundary reflection parameter $r$ to be negligible

$$
\begin{gathered}
\gamma_{f L}=1+\mu_{s}+\mu \frac{\left(1-\mu_{s}\right)}{(1-\mu)}+(\eta+1)+[F(\alpha)-A]^{-1} \\
\left\{X(\eta+1)+Y\left[-1-\mu \frac{\left(1-\mu_{s}\right)}{(1-\mu)}\right]\right\}
\end{gathered}
$$

2.2.3 The transverse gauge factor When the transverse strain $d w / w$ of the substrate is transmitted to the film we have

$$
\begin{aligned}
& \frac{d \lambda}{\lambda}=-\eta \frac{d w}{w} \\
& \frac{d t}{t}=-\mu \frac{\left(1-\mu_{s}\right)}{(1-\mu)} \frac{d w}{w}
\end{aligned}
$$

As the applied transverse strain introduces a change $d D / D$ in the grain size diameter of the form $d D / D \approx$ $d L / L$, we assume that

$$
\frac{d D}{D} \approx-\mu_{s} \frac{d w}{w}
$$

Finally the transverse gauge factor $\gamma_{f T}$ of the film becomes

$$
\begin{aligned}
\gamma_{f T}= & \mu \frac{\left(1-\mu_{s}\right)}{(1-\mu)}+\eta-\mu_{s}+[F(\alpha)-A]^{-1}\{X \\
& \left.\left(\eta-\mu_{s}\right)+Y\left[\mu_{s}-\mu \frac{\left(1-\mu_{s}\right)}{(1-\mu)}\right]\right\}
\end{aligned}
$$

\subsubsection{The grain-boundary transverse and longitudinal} gauge factors When the electronic scattering on external surfaces in assumed to be essentially specular (i.e. $p=1$ ) Eq. (20) and (22) reduce to

$$
\gamma_{g L}=1+\mu_{s}+\mu \frac{\left(1-\mu_{s}\right)}{(1-\mu)}+(1+\eta)\left\{1+\frac{G(\alpha)}{F(\alpha)}\right\}
$$

$$
\gamma_{g T}=\mu \frac{\left(1-\mu_{s}\right)}{(1-\mu)}+\left(\eta-\mu_{s}\right)\left\{1+\frac{G(\alpha)}{F(\alpha)}\right\}
$$

where $\gamma_{g L}$ and $\gamma_{g T}$ are the longitudinal and transverse gauge factor respectively.

These equations describe the effect of grainboundaries on the strain sensitivity of thin continuous films ${ }^{11,12}$. Note that Eq. (23) agrees with that derived by A. Singh ${ }^{12}$.

The values of the strain coefficients $\gamma_{g T}$ and $\gamma_{g L}$ of a polycrystalline film are constant with film thickness, $\gamma_{g T}^{g}$ and $\gamma_{g L}^{g}$ (calculated from Eqs. (23) and (24) with constant values of $\alpha$ ).

Variations in the polycrystalline film strain coefficients $\gamma_{g T}^{g}$ and $\gamma_{g L}^{g}$ are shown in Figures 1 and 2. In agreement with A. Singh ${ }^{12}$ we observe a marked dependence of the strain coefficients with the $\alpha$ values: they decrease with decreasing grain size $D$ and increasing grain-boundary reflection coefficient $r$.

For monocrystalline films, the variations in the gauge factors, respectively $\gamma_{g T}$ and $\gamma_{g L}$, are plotted versus the ratio $k$ of the film thickness $t$ to the bulk mean free path $\lambda$ for different values of the grain-

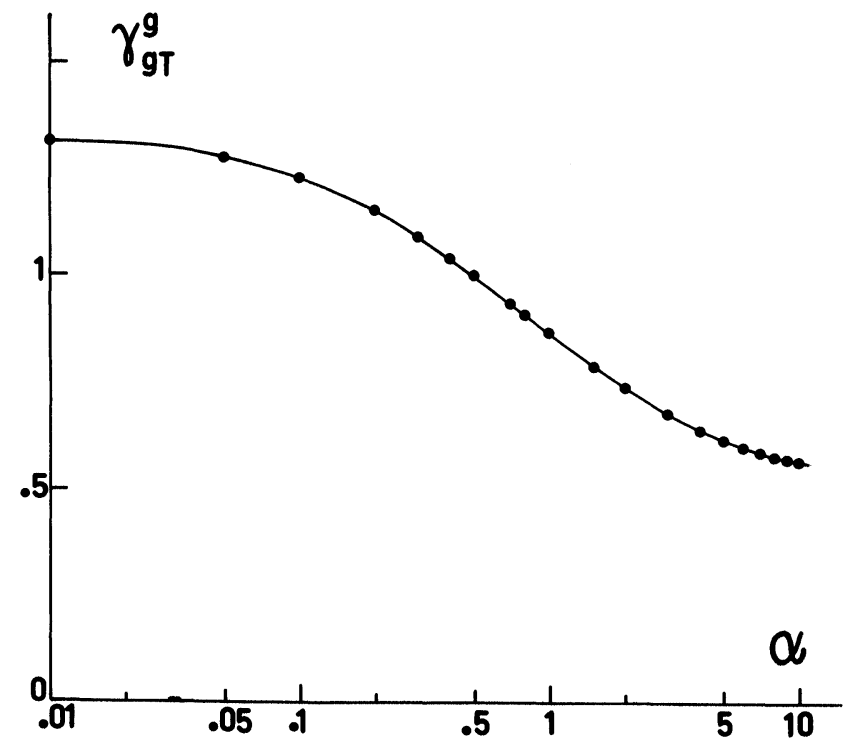

FIGURE 1 The transverse gauge factor $\gamma_{g T}^{g}$ of polycrystalline films versus the $\alpha$ parameter (from equation 1.4).

It has been assumed that the Gruneisen's constant is $g=2.705$ the Poisson's ratios of the substrate material and the film material are $\mu_{s}=0.25$ and $\mu=0.4$ respectively. 

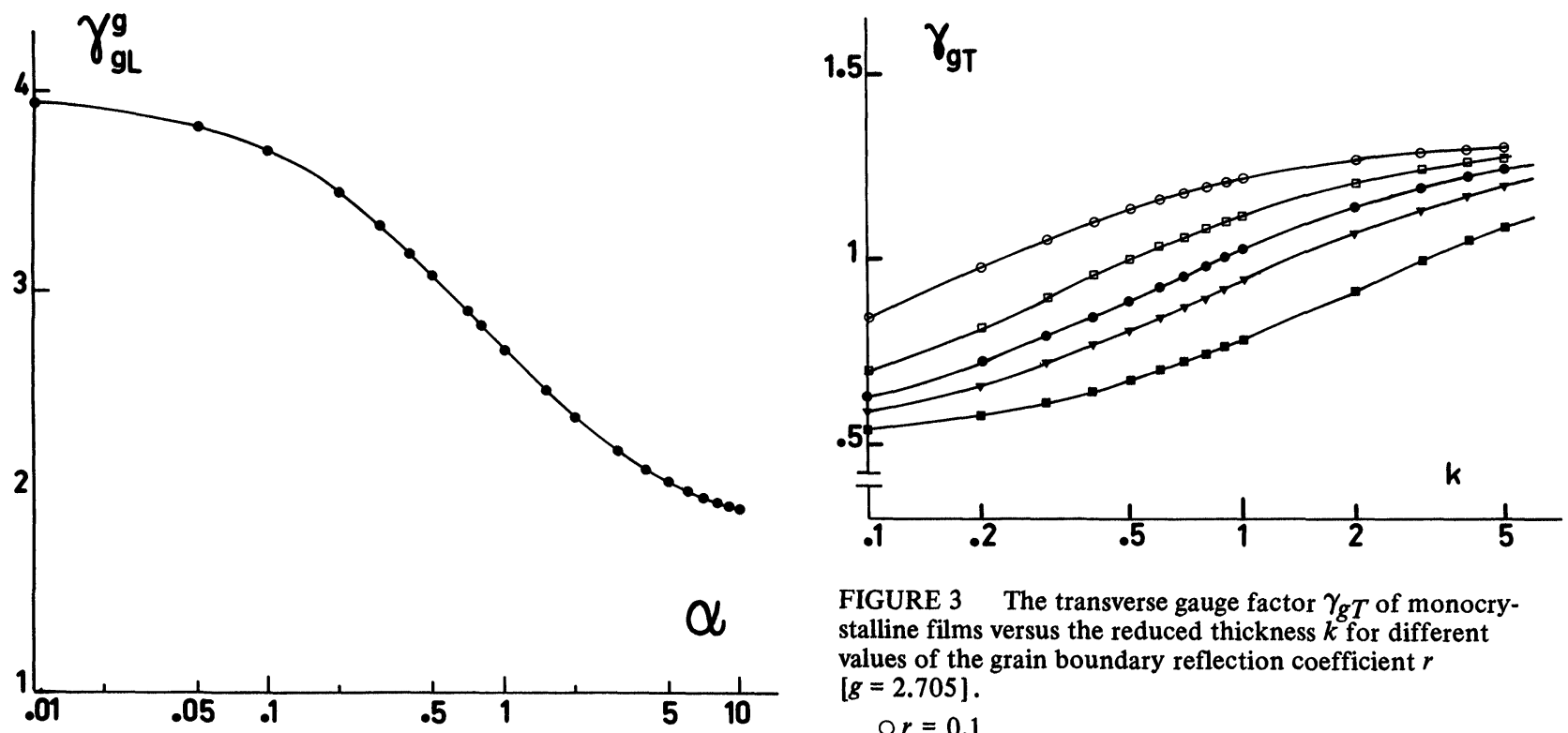

FIGURE 3 The transverse gauge factor $\gamma_{g T}$ of monocrystalline films versus the reduced thickness $k$ for different values of the grain boundary reflection coefficient $r$ $[g=2.705]$.
or $=0.1$
$\square r=0.2$
- $r=0.3$
$\nabla r=0.4$
$\square r=0.6$

boundary reflection coefficient $r$ (Figures 3 and 4). The effect of the grain-boundary reflection coefficient $r$ on the longitudinal strain coefficient, as shown on Figure 5, is marked.

It is reasonable to assume that infinitely thick polycrystalline or monocrystalline films are unsupported. Hence, the gauge factors of such films may be written:

$$
\begin{aligned}
& \gamma_{g L}^{\infty} \approx 1+2 \mu+(\eta+1)\left\{1+\frac{G(\alpha)}{F(\alpha)}\right\} \\
& \gamma_{g T}^{\infty} \approx \eta+(\eta-\mu) \cdot \frac{G(\alpha)}{F(\alpha)}
\end{aligned}
$$

For monocrystalline films the $\alpha$ parameter is observed to be a function of thickness $t$ and is found to be equal to 0 when the thickness becomes infinite. Thus, for an infinitely thick monocrystalline film it is easy to verify by making the substitution $\alpha=0$ into Eq. (25) and (26) that we obtain the theoretical bulk values of the strain coefficient, i.e.

$$
\gamma_{b L} \approx 2(1+\mu)+\eta
$$

and

$$
\gamma_{b T} \approx \eta
$$

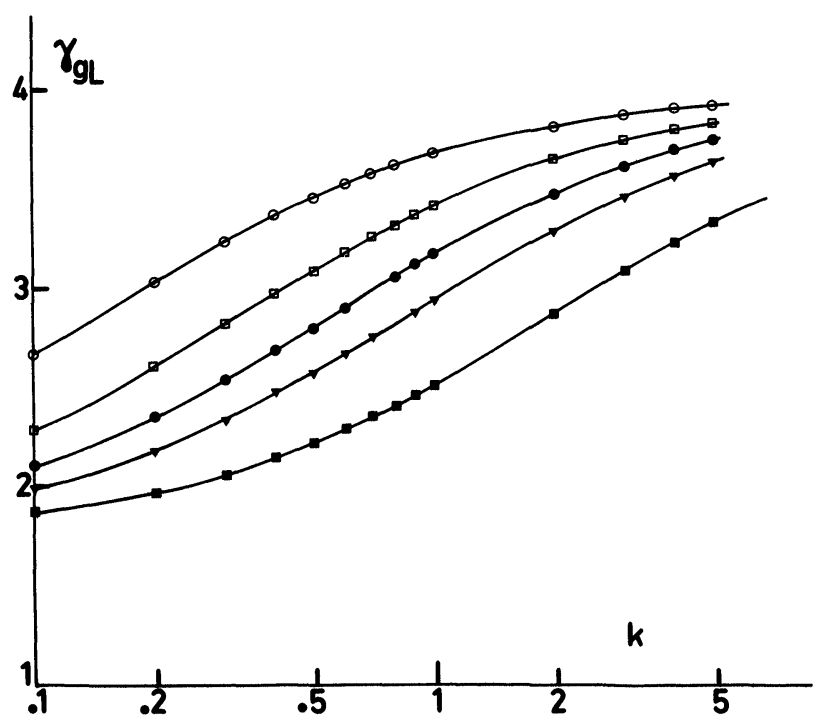

FIGURE 4 The longitudinal gauge factor $\gamma_{g L}$ of monocrystalline films versus the reduced thickness $k[g=3.08]$.

$$
\begin{aligned}
& \circ r=0.1 \\
& \square r=0.2 \\
& \bullet r=0.3 \\
& \nabla r=0.4 \\
& \square r=0.6
\end{aligned}
$$




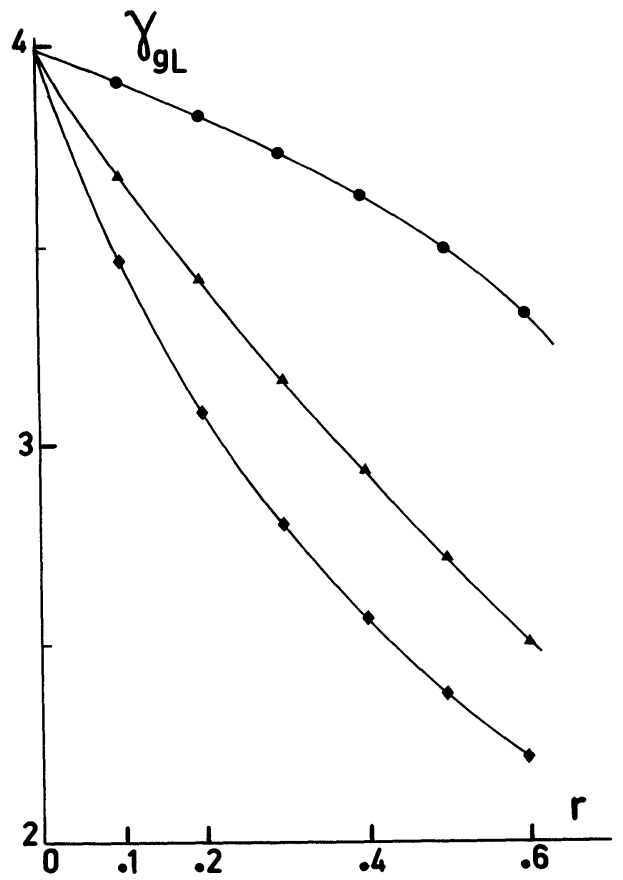

FIGURE 5 Dependence of the longitudinal strain coefficient $\gamma_{g L}$ on grain boundary reflection coefficient $r$ for different values of the reduced thickness $k$.

$$
\begin{aligned}
\bullet k & =0.5 \\
\Delta k & =1 \\
-k & =5
\end{aligned}
$$

For polycrystalline films, we may define the gauge factors

$$
\gamma_{g L}^{g \infty} \text { and } \gamma_{g T}^{g \infty}
$$

of infinitely thick film as

$$
\begin{array}{ll}
\gamma_{g L}^{g \infty}=\gamma_{g L}^{\infty} & \quad(\alpha \text { constant }) \\
\gamma_{g T}^{g \infty}=\gamma_{g T}^{\infty} & \quad(\alpha \text { constant })
\end{array}
$$

\subsubsection{The transverse and longitudinal gauge factors} of unsupported films For films in which the three types of electron scattering simultaneously occur (i.e. an isotropic background scattering, a grain boundary scattering and an external surface scattering) the general equations 20 and 22 may be rearranged by introducing the gauge factors of an infinitely thick films.

It yields, from Eqs. (20) and (22), in the case of monocrystalline films

$$
\begin{aligned}
\gamma_{f L} & =\gamma_{b L}+\mu_{s}-2 \mu+\mu \frac{1-\mu_{s}}{1-\mu} \\
& +[F(\alpha)-A]^{-1}\{X(\eta+1) \\
& \left.+Y\left[-1-\mu \frac{1-\mu_{s}}{1-\mu}\right]\right\} \\
\gamma_{f T} & =\gamma_{b T}+\mu \frac{1-\mu_{s}}{1-\mu}-\mu_{s}+[F(\alpha)-A]^{-1}\{X \\
& \left.\left(\eta-\mu_{s}\right)+Y\left(\mu_{s}-\mu \frac{1-\mu_{s}}{1-\mu}\right)\right\}
\end{aligned}
$$

For unsupported films Eqs. (27) and (28) reduce to the simple forms:

$$
\begin{aligned}
\gamma_{f L} & =\gamma_{b L}+[F(\alpha)-A]^{-1}\{X(\eta+1) \\
& -Y(\mu+1)\}
\end{aligned}
$$

and

$$
\gamma_{f T}=\gamma_{b T}+[F(\alpha)-A]^{-1} \quad\{X(\eta-\mu)\}
$$

This yields, in the case of polycrystalline films:

$$
\begin{aligned}
\gamma_{f L} & =\gamma_{g L}^{\infty}+\mu_{s}-2 \mu+\mu \frac{1-\mu_{s}}{1-\mu} \\
& +(\eta+1)\left[\frac{X}{F(\alpha)-A}-\frac{G(\alpha)}{F(\alpha)}\right]-Y[F(\alpha)-A]^{-1} \\
& \left\{1+\mu \frac{1-\mu_{s}}{1-\mu}\right\} \\
\gamma_{f T} & =\gamma_{g T}^{\infty}-\mu_{s}+\mu \frac{1-\mu_{s}}{1-\mu}-(\eta-\mu) \frac{G(\alpha)}{F(\alpha)} \\
& +[F(\alpha)-A]^{-1}\left\{X\left(\eta-\mu_{s}\right)+Y\left(\mu_{s}-\mu \frac{1-\mu_{s}}{1-\mu}\right)\right\}
\end{aligned}
$$

For unsupported films Eqs. (29) and (30) may be written:

$$
\begin{aligned}
\gamma_{f L} & =\gamma_{g L}^{\infty}+(\eta+1)\left[\frac{X}{F(\alpha)-A}-\frac{G(\alpha)}{F(\alpha)}\right]-Y \\
& \frac{1+\mu}{F(\alpha)-A} \\
\gamma_{f T} & =\gamma_{g T}^{\infty}+(\eta-\mu)\left[\frac{X}{F(\alpha)-A}-\frac{G(\alpha)}{F(\alpha)}\right]
\end{aligned}
$$


Eqs. (27) to (30) can be evaluated numerically with a digital computer.

We can compare the relations to those given by Singh $^{12}$ Eq. (13). According to the author, the longitudinal gauge factor of resistivity is

$$
\gamma=(\eta+1)\left[1+\frac{G(\alpha)}{F(\alpha)}\right]-\frac{F(\alpha)}{\frac{8}{3} k+F(\alpha)}(\eta-\mu)
$$

assuming that $p=o$ and $k \gg 1$.

Under these assumptions, Eqs. (18.1), (18.2) and (18.4) reduce to

$$
\begin{aligned}
& X \approx G(\alpha)-A^{\prime \prime}+A^{\prime} \\
& Y \approx-A^{\prime}
\end{aligned}
$$

where

$$
\begin{aligned}
& A^{\prime} \approx \frac{6}{\pi k} \int_{0}^{\pi / 2} d \phi \int_{1}^{\infty} \frac{\cos ^{2} \phi}{H^{2}(\tau, \phi)}\left[\frac{1}{\tau^{3}}-\frac{1}{\tau^{5}}\right] d \tau \\
& A^{\prime \prime} \approx \frac{12}{\pi k} \int_{0}^{\pi / 2} d \phi \int_{1}^{\infty} \frac{\cos ^{2} \phi}{H^{3}(\tau, \phi)}\left[\frac{1}{\tau^{3}}-\frac{1}{\tau^{5}}\right] d \tau
\end{aligned}
$$

For an unsupported polycrystalline film, the longitudinal gauge factor of resistivity is

$$
\gamma_{f L}^{\prime}=\gamma_{f L}-(1+2 \mu)
$$

Hence, Eqs. (32) and (33) yield

$$
\begin{aligned}
& \gamma_{f L}^{\prime} \approx(\eta+1)\left[i+\frac{G(\alpha)-A^{\prime \prime}+A^{\prime}}{F(\alpha)-A^{\prime}}\right] \\
& +\frac{A^{\prime}}{F(\alpha)-A^{\prime}}(1+\mu)
\end{aligned}
$$

This equation takes the form of Eq. (36)

$$
\begin{aligned}
& \gamma_{f L}^{\prime} \approx(\eta+1)\left[1+\frac{G(\alpha)+2 A^{\prime}-A^{\prime \prime}}{F(\alpha)-A^{\prime}}\right] \\
& +(\eta-\mu)\left[1-\frac{F(\alpha)}{F(\alpha)-A^{\prime}}\right]
\end{aligned}
$$

which qualitatively agrees with Eq. (31).

\section{PRACTICAL CONSIDERATIONS}

It is the purpose of this section to discuss the advantage or disadvantages of using as strain devices thin metal films whose transport parameters can be described by the Mayadas-Shatzkes model.

Theoretical relations show that relatively thick monocrystalline films have values of the gauge factors lower than those of the bulk material. This is in good agreement with the experimental results of authors ${ }^{5}$ who have studied the strain sensitivity of thin evaporated metal films with monocrystalline structure. It has been suggested that these results were probably due to the grain boundary scattering contribution to the resistivity; however when the grain boundary reflection coefficient $r$ remains lower than 0.2 , the difference between bulk and film gauge factor is about $10 \%$ in this range of thickness and no marked discrepancy could be observed. When the metallic layer is very thin, the change in electrical resistance with applied strain is strongly modified by both grain and external boundaries scatterings; for thin films whose structure is continuous even for reduced thicknesses equal to 0.5 , the ratio of the bulk to the film gauge factor decreases with increasing values of the boundary reflection coefficient $r$.

As the values of infinitely thick polycrystalline films gauge factors are similar to the $\gamma_{g}^{g}$ values it is easy to verify that values of thin polycrystalline film gauge factors are determined by the $\alpha$ parameter values. Then low values of gauge factors are expected for thin and even for relatively thick polycrystalline films which keep high values of the $\alpha$ parameter $(\alpha>3)$. It is instructive to evaluate the numerical values of longitudinal gauge factor for polycrystalline films related to differents values of the $\alpha$ parameter and reduced thickness $k$; when $\alpha=3$ and $k$ is equal to 0.5 and 5 respectively the ratio $\gamma_{f L} / \gamma_{b}$ becomes approximatively equal to 0.4 and 0.5 ; these values increase to 0.61 and 0.76 when $\alpha$ decreases to 0.5 . Finally we can summarize by observing that polycrystalline films exhibit poor strain transmission. From this point of view it seems that such films constitute a poor application for strain gauge in regard with other devices such as semiconductor ${ }^{28,29}$ or discontinuous ${ }^{5,30}$ metal films, for which several authors have measured gauge factor in the range $10-50^{28,29}$ or in the vicinity of $100^{5,30}$

However, in practice, the difficulty of obtaining reproducible and thermally stable thin film strain gauges has limited their application. In thin film strain gauge applications, the temperature stability, the reproducibility and reliability of operation are effectively of prime importance over a working temperature range.

Generally, ${ }^{27}$ the temperature coefficient of the gauge factor $\gamma$ is expressed as 


$$
\xi=\frac{1}{\gamma} \frac{\partial \gamma}{\partial T}
$$

If $\partial \epsilon$ is related to the change in strain $\epsilon$ (i.e. $\partial \epsilon \approx d L / L$ or $d w / w)$, we can write

$$
\begin{aligned}
\xi= & \left\{1 / \frac{1}{R_{f}}\left(\frac{\partial R_{f}}{\partial \epsilon}\right)_{T}\right\} \\
& {\left[\frac{\partial}{\partial T}\left\{\frac{1}{R_{f}}\left(\frac{\partial R_{f}}{\partial \epsilon}\right)_{T}\right\}\right]_{\epsilon} }
\end{aligned}
$$

As the temperature coefficient of resistance (T.C.R.) is defined by

$$
\beta=\frac{1}{R_{f}}\left(\frac{\partial R_{f}}{\partial T}\right)_{\epsilon}
$$

equation (39) gives $^{2}$

$$
\xi=-\beta+\frac{1}{R_{f}} \frac{\partial}{\partial T}\left(\frac{\partial R}{\partial \epsilon}\right)_{T}
$$

For metal films in the thickness range 300 to $1300 \AA$, Verma and Juretschtke ${ }^{3}$ have shown that $\partial R / \partial \epsilon$ is independent of temperature. Hence Eq. (39) reduces to $\xi \approx-\beta$.

This result is of great interest when considering the choice of the material to include in the strain device; for continuous thin metal film strain gauge, it is clear that a good temperature stability may be expected if the film material exhibits a low T.C.R.

Let us note that the T.C.R. $\beta_{g}^{g}$ of an infinitely thick polycrystalline film is related to the bulk T.C.R. $\beta_{b}$ by Eq. (40)

$$
\beta_{g}^{g} / \beta_{b}=1+\frac{G(\alpha)}{F(\alpha)}
$$

Values of the ratio $\beta_{g}^{g} / \beta_{b}$ versus $\alpha$ are reported on Figure 6 . The inspection of Figure 6 shows that the film T.C.R. decreases with increasing values of $\alpha$ and that, for example, a relatively thick polycrystalline film $(\alpha=5, k=5)$ having a gauge factor ratio $\gamma_{f} / \gamma_{b} \approx 0.5$ should have a T.C.R. ratio $\beta_{f} / \beta_{b}$ of about 0.11 , (instead of 0.8 for thin film in which Fuchs-Sondheimer conduction occurs).

It is not the purpose of this section to discuss the temporal stability of thin film strain gauges, but at this stage it is necessary to compare the thermal stability of different materials which constitute strain gauges. The exponential dependence of the carrier concentration in semiconductors films and in the same manner the semiconductor nature of activated

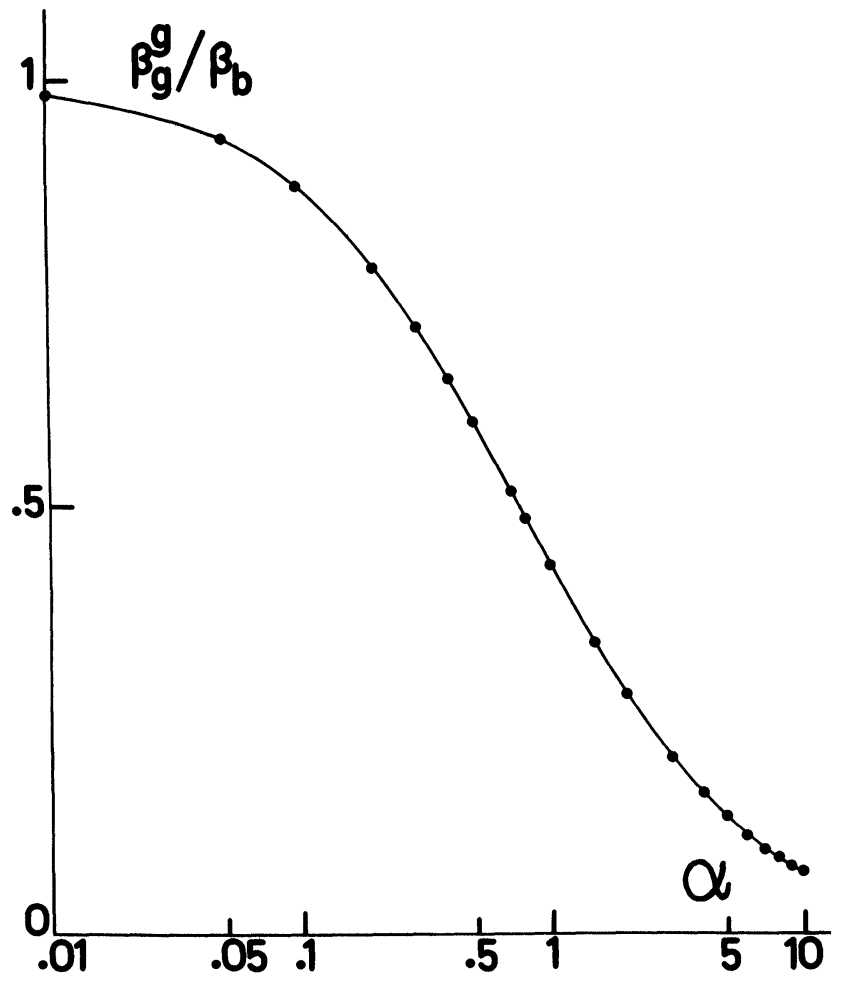

FIGURE 6 The reduced infinitely thick polycrystalline film T.C.R. $\beta_{g}^{g} / \beta_{b}$ versus the $\alpha$ parameter.

conduction process in discontinuous metal films should yield large negative values of the T.C.R. Consequently such films will exhibit very poor thermal stability and will require temperature compensation which may be achieved by using a thermistor or a temperature compensating Wheatstone bridge. On the contrary, in polycrystalline films which exhibit relatively large values of $\alpha$, the predicted low values of $\beta_{g}^{g}$ are effectively observed $\left(\approx 10^{-3} K^{-1}\right)^{31}$ and a better thermal stability is expected. This makes polycrystalline films interesting for strain gauge application even if we measure a low value of the gauge factor.

It is clear that monocrystalline films require a similar analysis.

\section{CONCLUSION}

Starting from Mayades-Shatzkes equations a theoretical study of the gauge factor of thin metallic films shows two main features: a poor strain transmission but a good thermal stability which depends on the 
technical procedure (grain size, grain-boundary reflection coefficient, ... .); this point should be extensively studied in the future.

\section{REFERENCES}

1. G. R. Witt, Thin Solid Films, 22 (1974) 133-156.

2. G. R. Witt, Thin Solid Films, 13 (1972) 109-115.

3. B. S. Verma and H. J. Juretschke, J. Appl. Phys., 41 (1970) 4732-4735.

4. C. Reale, Czech. J. phys. B, 21 (1971) 662-672.

5. R. L. Parker and A. Krinsky, J. Appl. Phys., 34 (1963) 2700-2708.

6. B. S. Verma and G. C. Jain, Thin Solid Films, 11 (1972) 27-32.

7. B. S. Verma and S. K. Sharma, Thin Solid Films, 5 (1970) R33 - R34.

8. Z. H. Meiksin and R. A. Hudzinski, J. Appl. Phys., 38 (1967) 4490-4494.

9. B. S. Verma and S. K. Sharma, Thin Solid Films, 5 (1970) R7 - R49.

10. B. S. Verma and S. K. Sharma, Thin Solid Films, 5 (1970) R44 - R46.

11. B. S. Verma, G. L. Malhotra and S. K. Sharma, Thin Solid Films, 6 (1970) R9 - R11.

12. Awatar Singh, Thin Solid Films, 21 (1974) 225-230.

13. K. L. Chopra, Thin Film Phenomena (McGraw-Hill, 1969) p 346.

14. C. Kooy and J. M. Nieuwen Huizen in R. Neidermayer and H. Mayer (Eds) Basic Problems in Thin Film Physics, Proc. Intern. Symp. Clausthal, Gottingen 1965, Vanderhoek and Ruprecht, Gottingen, 1966, p 181.
15. J. W. Geus in J. R. Anderson (Ed) Chemisorption and Reaction on Metallic Films, Academic Press, 1971, p 400.

16. E. E. Mola, J. Borrajo and J. M. Heras, Surface Sci., 34 (1973) 561-570.

17. A. F. Mayadas and M. Shatzkes, Phys. Rev. B., 1 (1970) $1382-1389$.

18. C. R. Tellier and A. J. Tosser, Thin Solid Films, 37 (1976) 207-214.

19. C. R. Tellier and A. J. Tosser, Thin Solid Films, 43 (1977) in the press.

20. C. R. Tellier and A. J. Tosser, Thin solid films, 35 (1976) 65-74.

21. B. Laville Saint Martin and G. Perny, Thin Solid Films, 4 (1969) 319.

22. B. Laville Saint Martin, Thin Solid Films, 5 (1970) 169-185.

23. A. J. Tosser, C. R. Tellier and J. Launey, International symposium on Vacuum and thin film Technology, Uppsala, Sweden (August 31 - September 3, 1976).

24. B. Singh, C. C. Ling and N. A. Surplice, Thin Solid Films, 24 (1974) 927-928.

25. A. K. Pal and S. Chaudhuri, J. Materials Sci., 11 (1976) 872-877.

26. Kh. M. Mannan and Kh R. Karim, J. Phys. F., 5 (1975) 1687-1693.

27. G. R. Witt and T. J. Coutts, Thin Solid Films, 7 (1971) R1.

28. M. Plassa and G. Rumiano, Thin Solid Films, 12 (1972) 75.

29. R. Koike, Elec. Eng. Japan, 84 (1964) 65.

30. M. J. Knight, J. Vac. Sci. \& Technol., 6 (1969) 706.

31. C. R. Tellier, A. J. Tosser, Thin Solid Films (to be published). 

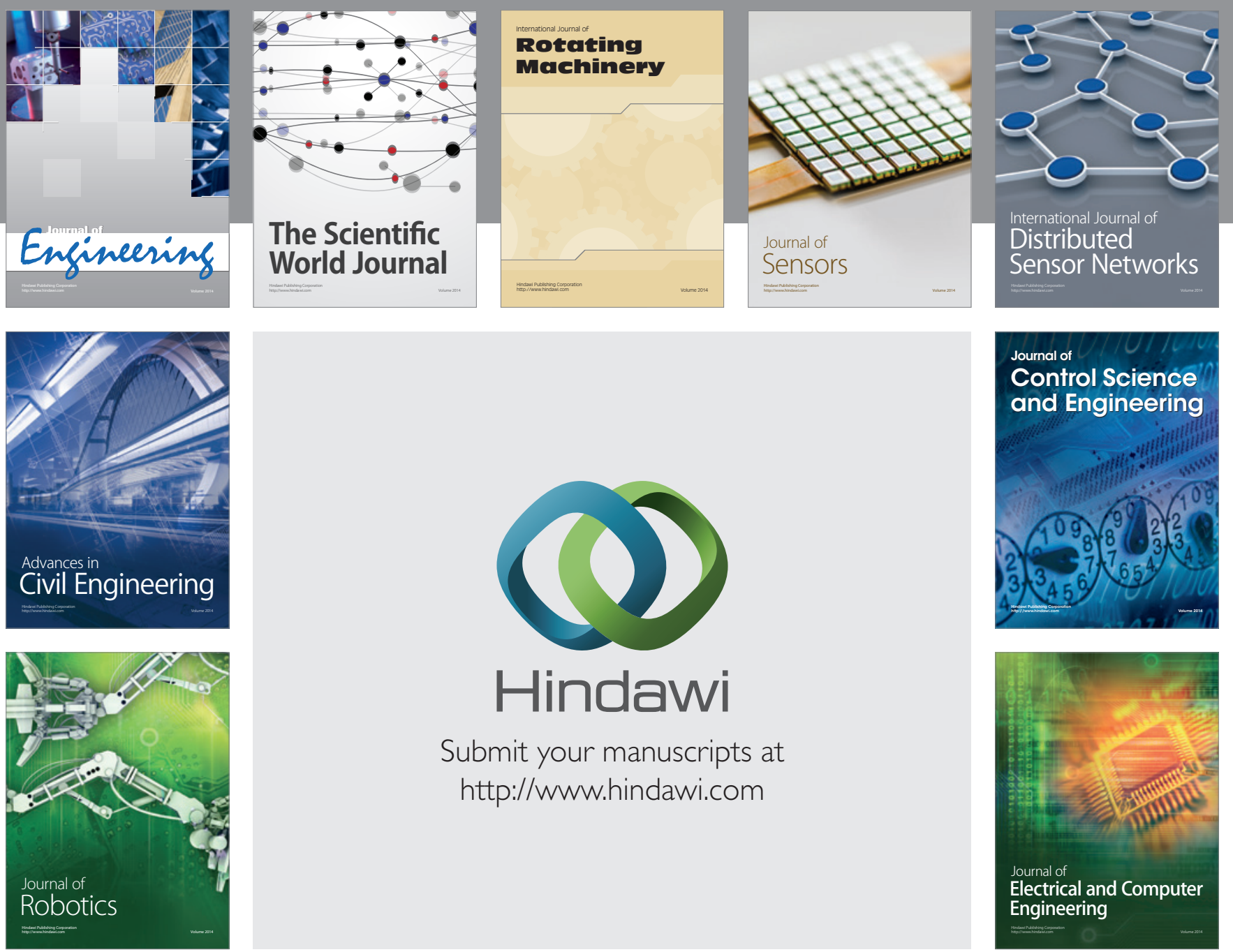

Submit your manuscripts at

http://www.hindawi.com
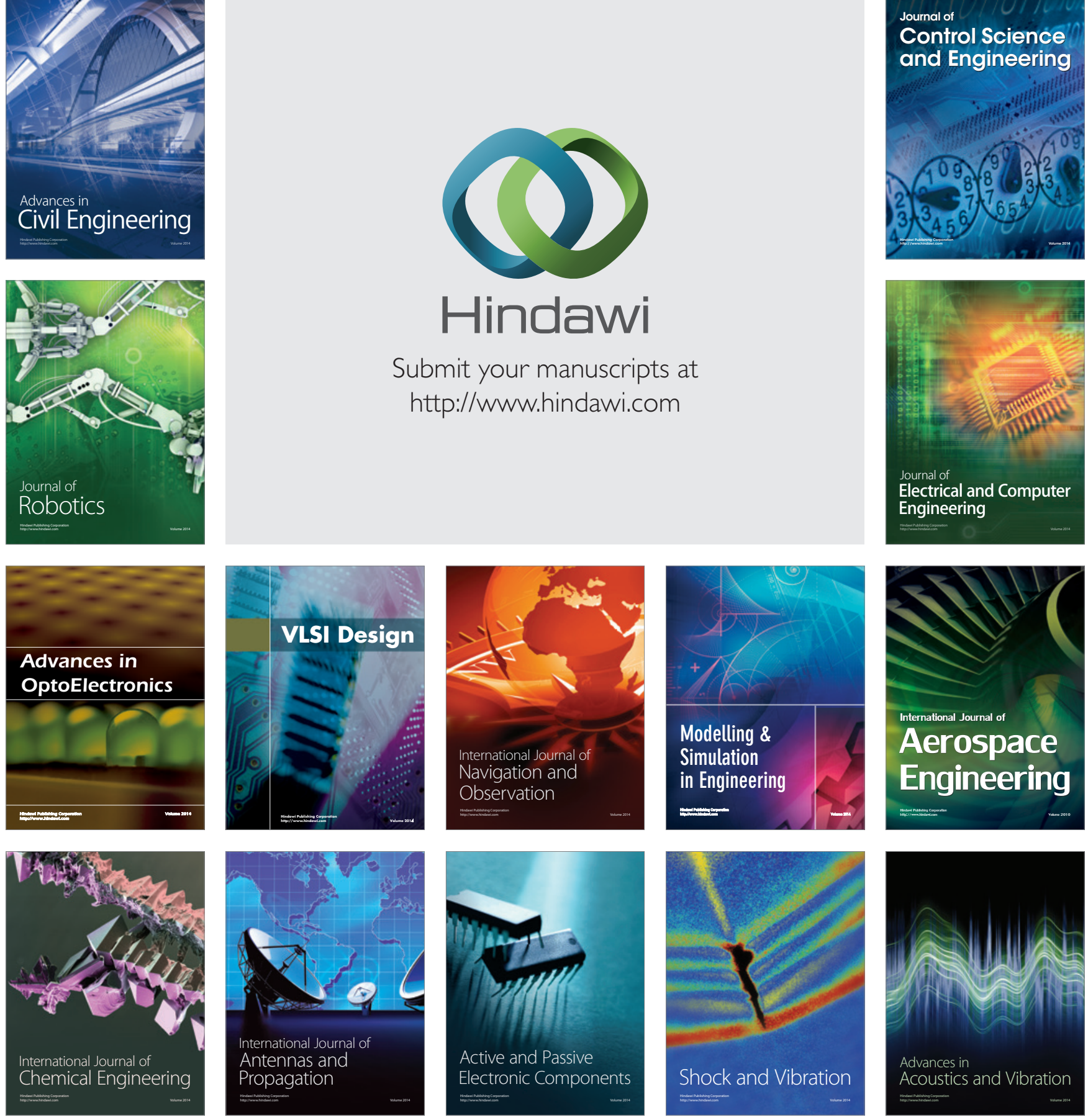